\title{
Mesenchymal Stem Cells and Cardiovascular Disease: A Bench to Bedside Roadmap
}

\author{
Manuel Mazo, Miriam Araña, Beatriz Pelacho, and Felipe Prosper \\ Department of Hematology and Cell Therapy, Clinica Universidad de Navarra, Foundation for Applied Medical Research, \\ University of Navarra, Avenida Pío XII 36, Pamplona, 31008 Navarra, Spain \\ Correspondence should be addressed to Felipe Prosper, fprosper@unav.es
}

Received 1 September 2011; Accepted 13 October 2011

Academic Editor: Wolfgang Wagner

Copyright ( $(2012$ Manuel Mazo et al. This is an open access article distributed under the Creative Commons Attribution License, which permits unrestricted use, distribution, and reproduction in any medium, provided the original work is properly cited.

In recent years, the incredible boost in stem cell research has kindled the expectations of both patients and physicians. Mesenchymal progenitors, owing to their availability, ease of manipulation, and therapeutic potential, have become one of the most attractive options for the treatment of a wide range of diseases, from cartilage defects to cardiac disorders. Moreover, their immunomodulatory capacity has opened up their allogenic use, consequently broadening the possibilities for their application. In this review, we will focus on their use in the therapy of myocardial infarction, looking at their characteristics, in vitro and in vivo mechanisms of action, as well as clinical trials.

\section{Introduction}

Although traditionally regarded as a health concern related particularly to the industrialized world, cardiovascular diseases are now the first cause of death worldwide [1], with myocardial infarction (MI) resulting in $12.8 \%$ of deaths. Aside from changes in ways of life associated with economic and social development, one of the main reasons is the fact that $\mathrm{MI}$ is an evolving disease. After the ischemic event, anaerobic conditions rapidly induce massive cell death, not only involving cardiomyocytes (CMs), but also vascular cells. Although the organism tries to exert a compensatory activity (reviewed in [2]) during the first stages of the disease and may even manage to partially restore functionality, the resulting scar is never repopulated, relentlessly leading the patient towards the setting of heart failure. Thus, though not conventionally regarded as such, cardiac disease is a degenerative affection in which lack of sufficient contractile and vascular cells leads to a decompensated neurohormonal microenvironment [3], which further impairs both organ function and cell survival.

Although the existence of stem cells has been a wellknown fact for nearly half a century [4], it is in the last 15 years that the field has experienced a major boost. Their capacity for differentiation has made stem cells outstanding candidates for the treatment of degenerative diseases, substituting for cells lost during the course of the disorder. Consequently, cardiac diseases and MI have been the object of intense research [5]. Among the cell types studied, mesenchymal stem cells (MSCs) are strong candidates for success in the MI setting. In the following pages, we will discuss their capacities as well as pre- and clinical investigations in which these cells have been employed.

\section{Origin, Types, and Characteristics}

The studies by Friedenstein and colleagues are regarded as one of the first reports on MSC [4]. In these, the clonogenic potential of a population of bone marrow- (BM-) derived stromal cells, described as colony-forming unit fibroblasts, was examined. BM is indeed one of the bestknown sources of progenitor cells, MSC being among them [6]. Although this is not entirely understood, BM-MSC are thought to act as supporters and nurturers of other cells within the marrow [7-9], possibly in a location close to blood vessels [10]. However, there is a relatively small population $(0.01 \%-0.0001 \%$ of nucleated cells in human BM [11]), so MSC can be easily purified by plastic adherence and expanded after BM extraction. Similarly, but adding 
simple mechanical and enzymatic processing, a mixed cell population (called stromal vascular fraction, SVF) can be isolated from adipose depots, which, after in vitro culture and homogenization, gives rise to the mesenchymal progenitors from this tissue, also termed adipose-derived stem cells (ADSCs) [12]. Adipose tissue is regarded as a much richer source of progenitors, harboring 100 to 500 times the numbers seen in BM [13]. However, despite similarities in phenotype, differentiation, or growth kinetics, there are certain differences at a functional, genomic, and proteomic level $[9,14]$, suggesting a degree of higher commitment of BM-MSC to chondrogenic and osteogenic lineages than ADSC [15].

Adipose tissue and BM are the most widely researched sources of mesenchymal progenitors because they are easy to harvest, and owing to the relative abundance of progenitors and the lack of ethical concerns. Nevertheless, MSCs have been ubiquitously found in a variety of locations, as umbilical cord blood [16], dental pulp [17], menstrual blood [18], or heart [19], among others (reviewed in [20]). This wide variety of origins, methodologies, and acronyms prompted standardization in 2005 by the International Society for Cellular Therapy, which set the minimum requirements for MSC definition (Table 1). First, MSC must be plasticadherent when maintained in standard culture conditions. Second, MSC must express CD105, CD73, and CD90, and lack expression of CD45, CD34, CD14 or CD11b, CD79a, or CD19 and HLA-DR surface molecules. Third, MSC must differentiate to osteoblasts, adipocytes, and chondroblasts in vitro [21]. Still, caution must be taken as some reports fail to meet these criteria, and MSC is often employed for "marrow stromal cell," "mesenchymal stromal cell" or "marrow stem cell." Accordingly, a clarification was published in which MSC was defined as "multipotent mesenchymal stromal Cells" [22], adding the supportive property to the required characteristics [23].

\section{What Do MSCs Have to Offer to Cardiac Regeneration?}

When considering the goal of cardiac tissue regeneration, the desired objective must encompass three objectives: (i) the production of a replacement myocardial mass, (ii) the formation of a functional vascular network to sustain it, and (iii) the returning of the impaired ventricle to its proper geometry. Cell therapy may theoretically affect those processes in two ways: either by direct differentiation of transplanted cells towards the desired lineages or by their production of molecules with therapeutic potential (Figure 1).

BM-MSC have shown their in vitro capacity to give rise to endothelial cells (ECs) [24, 25] and smooth muscle cells (SMCs) [24]. Cardiomyocyte differentiation has proved more problematic, as either demethylating agents have been employed [26], or it has been inefficient and incomplete $[27,28]$. In contrast, the cardiac potential of ADSC is better documented in vitro, showing their capacity to give rise to CM, either by the use of DMSO [29] or CM extracts [30]. In addition, ADSC seems to harbor a progenitor subset
TABLE 1: Standardized requirements for MSC definition.

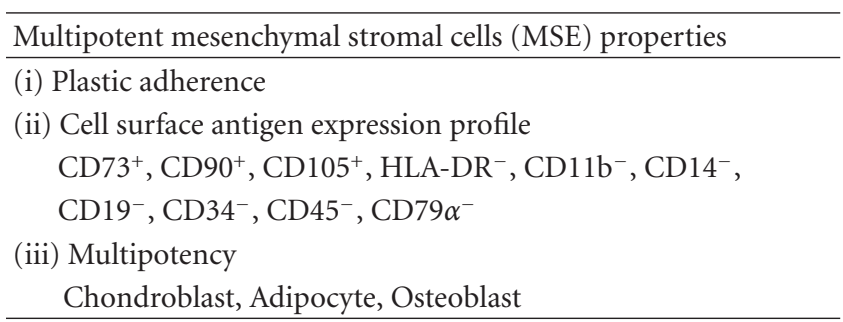

characterized by the expression of $\mathrm{Nkx} 2.5$ and $\mathrm{Mcl} 2 \mathrm{v}$ [31] and whose differentiation relies on the autocrine/paracrine activity of vascular endothelial growth factor (VEGF) [32]. SMC [33] and EC [34] have been obtained from adipose cells, yet a cautionary note must be struck, as some of these studies either rely on subpopulations of freshly isolated cells or culture them in differentiation-promoting medium before purifying the mesenchymal population [35, 36]. Finally, other mesenchymal progenitors have also been differentiated to $\mathrm{CM}$ or CM-like cells, such as menstrual blood-derived MSC [18] or umbilical cord blood MSC [37].

However, although it is extremely interesting, this differentiation potential must cope with two opposing factors. First, patients receiving stem cell therapy are severely diseased and usually elderly, two factors that have an outstanding impact on stem cell function. For instance, a decrease in the numbers and functionality of circulating endothelial progenitors is directly related to cardiovascular risks and smoking $[38,39]$ and age has also been shown to impair the angiogenic capacity of both ADSC [40] and BM-MSC [41]. Second, the small percentage of engrafted cells (see [42] for a review) coupled to the huge catastrophe caused by an MI (the loss in some cases of over 1 billion CM [43]) and the low rate of differentiation achieved even under in vitro controlled conditions makes the adding of such small number of cells a therapeutically inefficient approach.

Nevertheless, secretion of beneficial molecules has been demonstrated to be able to exert a positive effect, even when a few engrafted cells are left [44]. These molecules can induce a benefit either by increasing tissue perfusion, decreasing collagen deposition and fibrosis, enhancing hostcell survival, or attracting/regulating endogenous progenitors. Thus, Chen and coworkers compared the expression profile of BM-MSC and dermal fibroblasts [45], showing that mesenchymal progenitors secreted a higher amount of several molecules, including the potent proangiogenic cytokine VEGF or the chemotactic stromal derived factor1 (SDF-1). Conditioned medium from BM-MSC induced the recruitment of EC and macrophages, and improved wound healing. Moreover, it has recently been shown that serum-deprived BM-MSC acquire EC features and increase the release of VEGF or hepatocyte growth factor (HGF), another potent angiogenic molecule [46], both of which have been reported to be secreted by ADSC [32, 47, 48]. Moreover, Dr. March's group demonstrated that ADSCs have a pericytic nature and are able to form and stabilize functional vascular networks when mixed with endothelial 


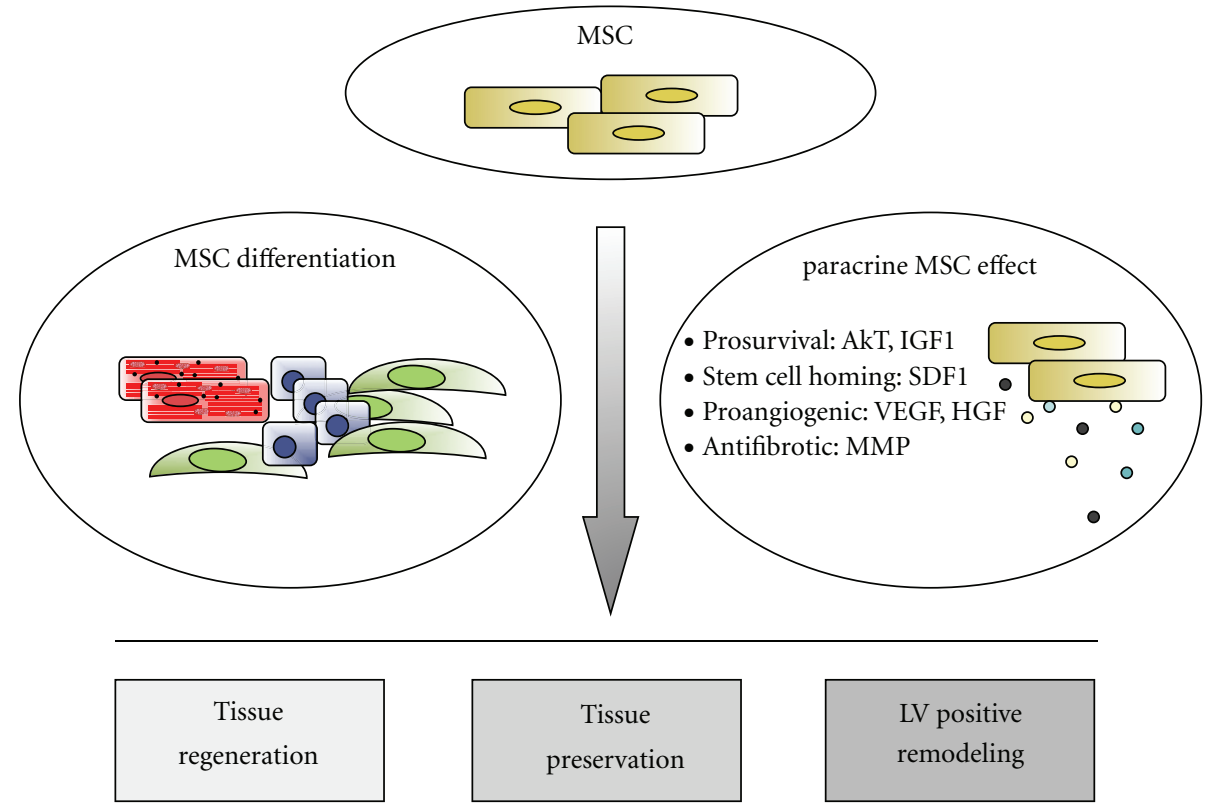

Figure 1: Main MSC actions on injured myocardium. Mesenchymal progenitors transplanted onto the ischemic myocardium are able to secrete a plethora of therapeutic molecules (paracrine activity) and even to differentiate towards (cardio-) vascular lineages, encouraging the healing of the damaged tissue, avoiding its transition to a scarred muscle, and regenerating the heart tissue mainly at the vascular level. Abbreviations: IGF-1: insulin-like growth factor-1; SDF-1: stromal derived factor-1; VEGF: vascular endothelial growth factor; HGF: hepatocyte growth factor; MMP: matrix metalloproteinase; LV: left ventricle.

progenitors [49]. Also, BM-MSC show a potent antifibrotic action, as their conditioned medium decreases cardiac fibroblast proliferation and expression of collagen types I and III $[50,51]$ and increases secretion of antifibrotic molecules such as matrix metalloproteinases (MMPs) 2, 9, and 14 [52]. These cells express five types of MMP $(2,13$ and membrane type-MMP 1,2, and 3) and are able to cross through type I collagen membranes [53], which theoretically would allow their trafficking across the infarction-derived scar. Likewise, ADSCs produce transforming growth factor- (TGF-) $\beta 1$ [54], a potent regulator of fibrosis. Taken as a whole, these examples demonstrate that mesenchymal progenitors are potent paracrine mediators with a considerable capacity to impact infarct evolution.

One last noteworthy competence is the ability of BMMSC and ADSC to modulate the immune response. Marrowderived mesenchymal progenitors inhibit the proliferation of activated $\mathrm{T}$ cells and the formation of cytotoxic $\mathrm{T}$ cells [55], inducing an anti-inflammatory phenotype, which would allow their allogenic use and significantly broaden the scope of their applicability. However, Huang et al. reported that differentiation reduced their capacity of immunological escape [56], related to an increase of immunostimulatory molecules MHC-Ia and II and a decrease in the immunosuppressive MHCIb. Along similar lines, McIntosh and coworkers reported that ADCS beyond passage one (and thus devoid of contaminating differentiated cells [57]) failed to elicit a response from allogenic $T$ cells [58], but this attribute may be diminished under inflammatory stimuli, as shown in vitro [59].
Finally, since the onset of induced pluripotent stem cells (iPSCs) [60], mesenchymal cells have been investigated [61, 62] due to their relatively easy harvest and higher potency than other cell types (e.g., dermal fibroblasts), which show an increased efficiency, even in the absence of the oncogene cMyc. Their supportive capacities have also made them good candidates to replace mouse cells as feeders $[63,64]$.

\section{MSC in Animal Models of MI}

However, in spite of all the positive characteristics of mesenchymal progenitors already depicted, their in vivo testing in animal models of the disease is compulsory. In this regard, three different settings can be found. First, the acute setting, in which cells are transplanted within hours of the MI. Here, the inflammatory microenvironment and the necrotic/apoptotic signals released from resident cells $[65$, 66] are the main opposing forces to the therapeutic activity of cells. Nevertheless, homing signals [67] and an antifibrotic milieu [68] may have a positive influence. Also, from a practical point of view, dealing with acute models offers the advantage of subjecting animals to only one surgery, as at the time of the MI (or minutes after it), the cells are applied, thus decreasing mortality and invasiveness. As a consequence, the majority of published reports use acute models [37, 6984]. Most studies (with the exception of the two by van der Bogt and colleagues [74, 77]) have consistently demonstrated that the treatment induces a significant benefit for cardiac function, mainly through paracrine mechanisms that induce an increase in tissue perfusion and a decrease in the size of the scar and collagen content. 
Similar results have been obtained in a second setting, the chronic one. Here, the repair processes that take place after ischemia have been completed, the scar has matured, and although a new network of blood vessels has been created, this is disorganized and inadequate $[85,86]$. These facts impose a great burden upon cell survival. However, it must be taken into account that the generation of homogeneous populations as BM-MSC or ADSC needs weeks of in vitro culture, thus, unless used in the allogeneic setting, there is no possibility of the bedside translation of the use of mesenchymal progenitors in the acute setting. In spite of this difficulty, fewer reports deal with this issue [87-90]. Compared to results in the acute setting, mesenchymal cell therapy of chronically infarcted hearts has a positive effect upon organ contractility and histology.

As a third and intermediate position, the so-called subacute model represents a situation where angiogenic processes are still on course, either through endothelial progenitors [91] or macrophages [92], and the receding of inflammation plus the increase in fibrotic processes are also on course. As with chronic models, there are few reports in this setting $[17,18,93,94]$, but again the benefit and mechanisms appear to be consistent.

Nevertheless, analyzing in more depth the studies mentioned above, it is possible to find a fair amount of information on how mesenchymal progenitors behave when injected into the diseased heart has been gathered. Chen et al. showed that transplantation of BM-MSC into chronically infarcted rabbit hearts induced an increase in the concentration of SDF-1 that elicited the chemotaxis of hostderived $\mathrm{BM}$ progenitors $\left(\mathrm{CD} 34^{+}, \mathrm{CD} 117^{+}, \mathrm{STRO}^{+}\right)$and was related to a functional benefit, a decrease in infarct size and improvement in tissue vascularization [89]. Li and coworkers demonstrated that the functional enhancement was accompanied by the augmented expression of the prosurvival gene Akt [95] whereas Mias and colleagues showed that the benefit upon contractility and remodeling in vivo was accompanied in vitro by a plethora of antifibrotic actions [52]. In a sheep model of MI, the group of Dr. Spinale monitored the evolution of MMP and their inhibitors, demonstrating a relationship with the number of transplanted cells [75]. Resembling their in vitro behavior, several publications have demonstrated the association between proangiogenic activity in vitro and secretion (either direct or host-derived) of angiogenic cytokines as VEGF, HGF, or insulin-like growth factor-1 (IGF-1), among others [17, 84, 93, 96, 97]. Whether these capacities are related to the claimed pericytic nature of these cells $[10,48,49]$ remains to be resolved.

Immune modulation (reviewed in [98]) in theory provides the means for the allogenic use of MSCs and as an off-the-shelf product (expanded prior to the onset of the ischemia and applicable on demand). Two reports have compared the effects of allogenic versus syngenic injection of BM-MSC in rat model of MI, with conflicting results. Imanishi et al. [78] demonstrated that both autologous and allogeneic cells improved cardiac function 4 weeks after transplantation, remained in the damaged tissues, and did not stimulate rejection. Huang and coworkers conversely [56] followed animals for up to 6 months. Syngenic cells stimulated cardiac recovery, but the effect of the allogenic treatment was transitory (significant 3 months after injection but not at 6) and BM-MSC disappeared earlier than their syngenic counterpart. However, this difference can be attributed to methodological discrepancies regarding time of transplantation (acute versus chronic resp.) or followup (1 versus 6 months). Equivalent and importantly, results from clinically relevant large animal models of MI in which allogenic cells have been employed have revealed either positive $[99,100]$ or no functional outcome [79]. In contrast, when autologous ADSC or BM-MSC are used [72, 83, $101,102]$, reports have shown a robust and consistent functional recovery after cell transplantation. Thus, strict considerations about building up animal models must be taken into account.

\section{Problems, Solutions}

Despite all the optimism, stem cell therapy shows certain caveats that are amenable to improvement, namely, lack of substantial engraftment and cell persistence, high levels of death, and low in vivo differentiation capacity. Some approaches to try to remedy these problems have included the use of genetic manipulation and in vitro pretreatment of cells or biomaterials. In this sense, the CXCR4/SDF-1 axis has been greatly exploited. Ma et al. investigated the peak of cardiac SDF-1 expression [103] in rat MI, finding that injected cells at that time point (1 day postinfarction) increased cell engraftment and tissue angiogenesis. Cheng and coworkers transplanted BM-MSC engineered to overexpress the receptor CXCR4, strengthening cell homing to the injured tissue after tail vein injection [104]. The same group combined BM-MSC peripheral injection with administration of granulocyte colony-stimulating factor, which in vitro increased CXCR4 expression. However, although engraftment was increased, no effect of cardiac function was found [105]. Huang and associates demonstrated that overexpression of the chemokine receptor CCR1 but not CXCR2 was associated with improved survival and grafting in a mouse model of MI, which also restored functionality [106].

Cell survival in the infarcted myocardium is jeopardized by hypoxia, inflammation, or oxidative stress. Liu et al. engineered BM-MSC to overexpress angiogenin [107], which improved hypoxic resistance in culture and was translated into an increase in cell engraftment and functional and histological recovery induction. Cell overexpression of hemeoxygenase-1 through adenoviral transfection showed superior therapeutic capacity, mainly through protection from inflammation and apoptosis [108], whereas targeted Akt overproduction in MSC restored cardiac function 2 weeks after MI through paracrine actions, including protection from hypoxia-induced apoptosis, release of cytokines, and preservation of tissue metabolism [109-111]. Others have explored antioxidants, like Song et al. who published that reactive oxygen species (ROS) diminished BM-MSC adherence to the substrate, but when treated with an ROS scavenger ( $\mathrm{N}$-acetyl-L-cysteine), engraftment was improved and the increase in fibrosis and infarct size prevented [112]. 
Hsp20 overexpression also protected MSC from oxidative stress and improved their beneficial activities [97].

However, viral or genetic modification of cells implies certain risks that currently make it difficult for a devised therapy to reach the bedside. Bioengineering uses biocompatible materials to improve or direct cell therapy and either synthetic or naturally derived systems have been employed. Jin and coworkers seeded BM-MSC on poly(lactide-co-1caprolactone) patches which when applied on a rat cryoinjury model were able to improve cardiac function and decrease infarct size [113]. Porcine small intestine submucosa, a decellularized substrate, has been employed to treat a rabbit model of chronic MI, showing a significant benefit upon contractility and histology, as well as cell migration towards the injured tissue [114]. The cell sheet technology allows increasing thickness through stacking of constructs, as shown by Chen et al. [115], where its transplantation in a rat syngenic model of cardiac ischemia improved cardiac function as well as paracrine secretion of therapeutic molecules by grafted cells. Dr. Mori's group compared the transplantation of a cell sheet seeded with ADSC versus fibroblasts, showing the superior effect of the mesenchymal progenitors [116]. Recently, autologous ADSC were transplanted along with allogenic ESC-derived $\mathrm{CD} 5^{+}$cardiac progenitors in a monkey model of infarction, demonstrating the safety of the procedure, although the functional outcome was not analyzed [117].

Finally, a word of caution must be added. Animal models of the disease are a powerful tool to explore the feasibility of a certain therapy, as MSC treatment of MI, but despite positive and reproducible results, rodent and even large animal models are just oversimplifications of the more complex setting of the human disease. As above stated, animals where cell therapy is applied are not elderly, nor severely diseased, thus making any result, even if tremendously positive, just a clue or hint before proceeding to the final application to patients, where the real safety and effectiveness can be assessed.

\section{Mesenchymal Progenitors and Clinical Application}

Several clinical trials have been performed with autologous BM-MSC, proving their safety when transplanted in patients with either acute or chronic myocardial infarction [118120]. Moreover, the first clinical trial designed as a randomized study showed an improvement in the cardiac func tion 3 months after BM-MSC intracoronary infusion in patients with acute MI [120]. In view of the encouraging results of the previous clinical trials, new phase-I/II studies have been initiated, including the transendocardial autologous cells (hMSC or hBMC) in Ischemic Heart Failure Trial (TAC-HFT; http://www.clinicaltrials.org/ NCT00768066/), the Prospective Randomised study Of MSC THErapy in patients Undergoing cardiac Surgery (PROMETHEUS) trial (http://www.clinicaltrials.org/ NCT00587990/), and the Percutaneous Stem Cell Injection Delivery Effects on Neomyogenesis (POSEIDON) pilot study
(http://www.clinicaltrials.org/NCT01087996/) [121], among others.

BM-MSCs from allogeneic origin have been tested as an off-the-shelf cell product. The first phase-I, randomized, double-blind, placebo-controlled, dose-escalation study was performed in 53 patients with acute MI, who intravenously received one of three doses of BM-MSCs $(0.5,1.6$ or $5.0 \times$ $10^{6} \mathrm{BM}-\mathrm{MSC} / \mathrm{Kg}$ body weight) derived from a single cell donor (Prochymal; Osiris therapeutics, Inc.) or placebo [122]. Safety of the procedure was proven, showing fewer episodes of ventricular tachycardia and even a better lung function in the cell-treated group. Also, renal, hepatic, and hematologic laboratory indexes were similar in the two groups and no patient developed tumors. Importantly, a significant increase was detected in the ejection fraction (EF) of the treated patients. In a magnetic resonance imaging substudy, cell treatment, but not placebo, increased left ventricular ejection fraction and led to a reversal of adverse remodeling after 6 months of treatment. Now, a phase-II multicentre trial of ProchymalTM has been started (http://www.clinicaltrials.org/NCT00877903/).

Furthermore, BM-MSC safety has been tested in patients with moderate-to-severe chronic heart failure in a phase-II, randomized, single-blind, placebo-controlled, dose-escalation, multicenter study. In this clinical trial, the patients received an endoventricular injection of an allogeneic BM-MSC product (Revascor, Mesoblast Ltd.) along the infarct border zone and no procedure-related complications were reported. Analysis of the data obtained after 6 months of followup (http://www.mesoblast.com/newsroom/asxannouncements/archives/) showed a significant decrease in the number of patients who developed any severe or major adverse cardiac event, such as composite of cardiac death, heart attack, or need for coronary revascularization procedures. Moreover, the first cohort in the study $(n=20$ patients), which received the low dose of the cell treatment, showed a significantly greater increase in the EF when compared with the control group [123].

On the other hand, regarding other sources of MSC such as adipose tissue, no clinical trials have been initiated yet, despite the fact that the beneficial potential of ADSC has been preclinically demonstrated [83]. Until now, only the noncultured adipose stromal vascular fraction is being tested at the clinical level. The first study, a double-blind, placebo-controlled trial named APOLLO (http://www.clinicaltrials.org/NCT00442806/; [124]) where AMI patients received autologous adipose derived stem cells by intracoronary infusion, was proven safe. Now, a phase II/III ADVANCE trial has been initiated to evaluate their efficacy (http://www.clinicaltrials.org/NCT01216995/).

In general, the results obtained from the many clinical trials performed, either with MSC or other stem cell populations (mainly BM-derived cells and skeletal myoblasts), have taught us several important lessons that will help to design and interpret the following clinical trials. (i) Cell treatment is not equally efficacious in all the patients. In general, it seems that the worse the heart damage (meaning severely decreased postrevascularization LVEF or high degree of infarct transmurality), the better the benefit induced by the 
transplanted cells seems to be [125-127]. (ii) Cell dose and timing for treatment are critical. Thus, a meta-analysis of the results obtained in the most relevant clinical trials performed in acute MI patients treated with BM cells has shown a significantly greater effect in those patients that received high cell doses $\left(10^{8}\right.$ cells). Also, the same study showed a greater beneficial effect when cells are infused during the first week after the infarct [128]. (iii) Autologous treatment is not necessarily the best. Until now, most of the clinical studies have been designed for autologous cell application in order to avoid the immunorejection of the transplanted cells. However, it has to be borne in mind that stem cells derived from aged patients with risk of atherosclerosis or other diseases might be defective, and thereby, treatment with them might not be as efficacious as with cells derived from young healthy donors [129-131]. In that sense, the use of MSC, which present immunomodulatory properties [132], could be of great relevance. Thus, advantages of allogeneic MSC treatment would be that, together with the putative greater paracrine effect that allogeneic cells derived from a healthy donor could exert, a fully tested clinical grade ready to use allogeneic cell product could be available for any patient. Importantly, patients with acute MI could also be eligible for such treatment. Furthermore, the logistical complexity and manufacturing costs that autologous cell preparation implies would be significantly reduced by the allogeneic application. However, caution should be taken when taking into consideration the issues related to their immune privilege explained above.

Thus, although it is mandatory to better understand the mechanisms involved in the MSC phenotype switch and to elucidate how this could affect the cells' potential benefit, it has to be considered that, in any case, because MSC would not differentiate towards cardiovascular cells and would act as a paracrine factor source [111], their permanent presence in the heart might not be necessary for therapeutic purposes. In that case, a temporarily action should be sufficient for exerting their benefit. Phase-II clinical trials are currently assessing the efficacy of the allogeneic MSC treatment, together with the long-term safety. If allogeneicity of the cells diminishes their effectiveness, several options could be considered, like temporal patient immunosuppression and/or donor-recipient HLA-II mismatch minimizing. As a consequence, the increase in the rate of engraftment of transplanted cells is so far one of the main challenges. As already indicated, the use of scaffolds could improve this factor. Interestingly, a clinical trial has been performed in 15 patients with chronic MI who were treated with a collagen scaffold previously seeded with bone marrow mononuclear cells [133]. The cellularized patch was implanted onto the pericardium and no adverse events were reported, showing the feasibility and safety of the treatment. Furthermore, a limiting effect in ventricular wall remodeling and an improved diastolic function were detected. These positive results will probably promote new larger randomized controlled trials, where mesenchymal and other stem cell populations might be tested in combination with scaffolds, thus leading to a further step in the therapeutic use of stem cells.

\section{Conclusion}

Mesenchymal cells have raised substantial interest in recent years due to their potential and versatility. Although we are only now starting to understand the mechanisms by which they repair or induce the repair of damaged organs, their pleiotropic activity and the technical ease of manipulation makes them good candidates for the treatment of the MI. Though waiting for randomized, double-blinded, placebocontrolled clinical trials in which large cohorts of patients could participate, the available data demonstrates the safety of the therapy and points towards a positive effect, further encouraging new investigations. The addition of the latest improvements in the field, including in vitro conditioning and bioengineering, will surely suppose a further step towards finding an optimized treatment. However, certain issues, mainly immunomodulatory capacity and allogenic use, need to be better understood.

\section{Acknowledgments}

This work was supported by grants to FP (FP7 INELPY, Instituto de Salud Carlos III (ISCIII-RETIC RD06/0014 (FIS)) and Ministero de Ciencia e Innovación Programa de Internacionalización CARDIOBIO) and BP (Instituto de Salud Carlos III (PI10/01621 and CP09/00333 (FIS)).

\section{References}

[1] World Health Organization, World Health Statistics 2008, 2008.

[2] M. Mazo, B. Pelacho, and F. Prósper, "Stem cell therapy for chronic myocardial infarction," Journal of Cardiovascular Translational Research, vol. 3, no. 2, pp. 79-88, 2010.

[3] N. G. Frangogiannis, "Chemokines in the ischemic myocardium: from inflammation to fibrosis," Inflammation Research, vol. 53, no. 11, pp. 585-595, 2004.

[4] A. J. Friedenstein, K. V. Petrakova, A. I. Kurolesova, and G. P. Frolova, "Heterotopic of bone marrow. Analysis of precursor cells for osteogenic and hematopoietic tissues," Transplantation, vol. 6, no. 2, pp. 230-247, 1968.

[5] B. Pelacho and F. Prosper, "Stem cells and cardiac disease: where are we going?" Current Stem Cell Research \& Therapy, vol. 3, no. 4, pp. 265-276, 2008.

[6] C. Clavel and C. M. Verfaillie, "Bone-marrow-derived cells and heart repair," Current Opinion in Organ Transplantation, vol. 13, no. 1, pp. 36-43, 2008.

[7] T. Walenda, S. Bork, P. Horn et al., "Co-culture with mesenchymal stromal cells increases proliferation and maintenance of haematopoietic progenitor cells," Journal of Cellular and Molecular Medicine, vol. 14, no. 1-2, pp. 337-350, 2010.

[8] D. L. Jones and A. J. Wagers, "No place like home: anatomy and function of the stem cell niche," Nature Reviews Molecular Cell Biology, vol. 9, no. 1, pp. 11-21, 2008.

[9] W. Wagner, C. Roderburg, F. Wein et al., "Molecular and secretory profiles of human mesenchymal stromal cells and their abilities to maintain primitive hematopoietic progenitors," Stem Cells, vol. 25, no. 10, pp. 2638-2647, 2007.

[10] X. Cai, Y. Lin, C. C. Friedrich et al., "Bone marrow derived pluripotent cells are pericytes which contribute to vascularization," Stem Cell Reviews and Reports, vol. 5, no. 4, pp. 437445, 2010. 
[11] M. F. Pittenger, A. M. Mackay, S. C. Beck et al., "Multilineage potential of adult human mesenchymal stem cells," Science, vol. 284, no. 5411, pp. 143-147, 1999.

[12] M. Mazo, J. J. Gavira, B. Pelacho, and F. Prosper, "Adiposederived stem cells for myocardial infarction," Journal of Cardiovascular Translational Research, vol. 4, no. 2, pp. 145153, 2011.

[13] L. Casteilla, V. Planat-Benard, P. Laharrague, and B. Cousin, "Adipose-derived stromal cells: their identity and uses in clinical trials, an update," World Journal of Stem Cells, vol. 3, pp. 25-33, 2011.

[14] S. Kern, H. Eichler, J. Stoeve, H. Kluter, and K. Bieback, "Comparative analysis of mesenchymal stem cells from bone marrow, umbilical cord blood, or adipose tissue," Stem Cells, vol. 24, no. 5, pp. 1294-1301, 2006.

[15] J. M. Gimble, A. J. Katz, and B. A. Bunnell, "Adipose-derived stem cells for regenerative medicine," Circulation Research, vol. 100, no. 9, pp. 1249-1260, 2007.

[16] S. E. Yang, C. W. Ha, M. H. Jung et al., "Mesenchymal stem/progenitor cells developed in cultures from UC blood," Cytotherapy, vol. 6, no. 5, pp. 476-486, 2004.

[17] C. Gandia, A. N.A. Armiñan, J. M. García-Verdugo et al., "Human dental pulp stem cells improve left ventricular function, induce angiogenesis, and reduce infarct size in rats with acute myocardial infarction," Stem Cells, vol. 26, no. 3, pp. 638-645, 2008.

[18] N. Hida, N. Nishiyama, S. Miyoshi et al., "Novel cardiac precursor-like cells from human menstrual blood-derived mesenchymal cells," Stem Cells, vol. 26, no. 7, pp. 1695-1704, 2008.

[19] S. Carlson, J. Trial, C. Soeller, and M. L. Entman, "Cardiac mesenchymal stem cells contribute to scar formation after myocardial infarction," Cardiovascular Research, vol. 91, no. 1, pp. 99-107, 2011.

[20] D. C. Ding, W. C. Shyu, and S. Z. Lin, "Mesenchymal stem cells," Cell Transplant, vol. 20, pp. 5-14, 2011.

[21] M. Dominici, K. Le Blanc, I. Mueller et al., "Minimal criteria for defining multipotent mesenchymal stromal cells. The International Society for Cellular Therapy position statement," Cytotherapy, vol. 8, no. 4, pp. 315-317, 2006.

[22] E. M. Horwitz, K. Le Blanc, M. Dominici et al., "Clarification of the nomenclature for MSC: the international society for cellular therapy position statement," Cytotherapy, vol. 7, no. 5, pp. 393-395, 2005.

[23] B. Sacchetti, A. Funari, S. Michienzi et al., "Self-renewing osteoprogenitors in bone marrow sinusoids can organize a hematopoietic microenvironment," Cell, vol. 131, no. 2, pp. 324-336, 2007.

[24] T. P. Lozito, J. M. Taboas, C. K. Kuo, and R. S. Tuan, "Mesenchymal stem cell modification of endothelial matrix regulates their vascular differentiation," Journal of Cellular Biochemistry, vol. 107, no. 4, pp. 706-713, 2009.

[25] J. W. Liu, S. Dunoyer-Geindre, V. Serre-Beinier et al., "Characterization of endothelial-like cells derived from human mesenchymal stem cells," Journal of Thrombosis and Haemostasis, vol. 5, no. 4, pp. 826-834, 2007.

[26] W. Xu, X. Zhang, H. Qian et al., "Mesenchymal stem cells from adult human bone marrow differentiate into a cardiomyocyte phenotype in vitro," Experimental Biology and Medicine, vol. 229, no. 7, pp. 623-631, 2004.

[27] X. Yan, A. Lv, Y. Xing et al., "Inhibition of p53-p21 pathway promotes the differentiation of rat bone marrow mesenchymal stem cells into cardiomyocytes," Molecular and Cellular Biochemistry, vol. 354, no. 1-2, pp. 21-28, 2011.
[28] A. Armiñán, C. Gandía, J. M. García-Verdugo et al., "Cardiac transcription factors driven lineage-specification of adult stem cells," Journal of Cardiovascular Translational Research, vol. 3, no. 1, pp. 61-65, 2010.

[29] A. van Dijk, H. W. M. Niessen, B. Zandieh Doulabi, F. C. Visser, and F. J. Van Milligen, "Differentiation of human adipose-derived stem cells towards cardiomyocytes is facilitated by laminin," Cell and Tissue Research, vol. 334, no. 3, pp. 457-467, 2008.

[30] K. G. Gaustad, A. C. Boquest, B. E. Anderson, A. M. Gerdes, and P. Collas, "Differentiation of human adipose tissue stem cells using extracts of rat cardiomyocytes," Biochemical and Biophysical Research Communications, vol. 314, no. 2, pp. 420-427, 2004.

[31] X. Bai, K. Pinkernell, Y. H. Song, C. Nabzdyk, J. Reiser, and E. Alt, "Genetically selected stem cells from human adipose tissue express cardiac markers," Biochemical and Biophysical Research Communications, vol. 353, no. 3, pp. 665-671, 2007.

[32] Y. H. Song, S. Gehmert, S. Sadat et al., "VEGF is critical for spontaneous differentiation of stem cells into cardiomyocytes," Biochemical and Biophysical Research Communications, vol. 354, no. 4, pp. 999-1003, 2007.

[33] Y. M. Kim, E. S. Jeon, M. R. Kim, S. K. Jho, S. W. Ryu, and J. H. Kim, "Angiotensin II-induced differentiation of adipose tissue-derived mesenchymal stem cells to smooth muscle-like cells," International Journal of Biochemistry and Cell Biology, vol. 40, no. 11, pp. 2482-2491, 2008.

[34] V. Planat-Benard, J. S. Silvestre, B. Cousin et al., "Plasticity of human adipose lineage cells toward endothelial cells: physiological and therapeutic perspectives," Circulation, vol. 109, no. 5, pp. 656-663, 2004.

[35] L. J. Fischer, S. McIlhenny, T. Tulenko et al., "Endothelial differentiation of adipose-derived stem cells: effects of endothelial cell growth supplement and shear force," Journal of Surgical Research, vol. 152, no. 1, pp. 157-166, 2009.

[36] C. Sengenès, A. Miranville, M. Maumus, S. De Barros, R. Busse, and A. Bouloumié, "Chemotaxis and differentiation of human adipose tissue CD34 +/CD31- progenitor cells: role of stromal derived factor- 1 released by adipose tissue capillary endothelial cells," Stem Cells, vol. 25, no. 9, pp. 2269-2276, 2007.

[37] S. A. Chang, J. L. Eun, H. J. Kang et al., "Impact of myocardial infarct proteins and oscillating pressure on the differentiation of mesenchymal stem cells: effect of acute myocardial infarction on stem cell differentiation," Stem Cells, vol. 26, no. 7, pp. 1901-1912, 2008.

[38] T. Kondo, M. Hayashi, K. Takeshita et al., "Smoking cessation rapidly increases circulating progenitor cells in peripheral blood in chronic smokers," Arteriosclerosis, Thrombosis, and Vascular Biology, vol. 24, no. 8, pp. 1442-1447, 2004.

[39] M. Vasa, S. Fichtlscherer, A. Aicher et al., "Number and migratory activity of circulating endothelial progenitor cells inversely correlate with risk factors for coronary artery disease," Circulation research, vol. 89, no. 1, pp. E1-7, 2001.

[40] R. Madonna, F. V. Renna, C. Cellini et al., "Age-dependent impairment of number and angiogenic potential of adipose tissue-derived progenitor cells," European Journal of Clinical Investigation, vol. 41, no. 2, pp. 126-133, 2011.

[41] H. Liang, H. Hou, W. Yi, G. Yang, C. Gu, W. B. Lau et al., "Increased expression of pigment epithelium-derived factor in aged mesenchymal stem cells impairs their therapeutic efficacy for attenuating myocardial infarction injury," European Heart Journal, In press. 
[42] H. K. Haider and M. Ashraf, "Strategies to promote donor cell survival: combining preconditioning approach with stem cell transplantation," Journal of Molecular and Cellular Cardiology, vol. 45, no. 4, pp. 554-566, 2008.

[43] T. E. Robey, M. K. Saiget, H. Reinecke, and C. E. Murry, "Systems approaches to preventing transplanted cell death in cardiac repair," Journal of Molecular and Cellular Cardiology, vol. 45, no. 4, pp. 567-581, 2008.

[44] P. W. M. Fedak, "Paracrine effects of cell transplantation: modifying ventricular remodeling in the failing heart," Seminars in Thoracic and Cardiovascular Surgery, vol. 20, no. 2, pp. 87-93, 2008.

[45] L. Chen, E. E. Tredget, P. Y. G. Wu, Y. Wu, and Y. Wu, "Paracrine factors of mesenchymal stem cells recruit macrophages and endothelial lineage cells and enhance wound healing," PLoS ONE, vol. 3, no. 4, Article ID e1886, 2008.

[46] A. Oskowitz, H. McFerrin, M. Gutschow, M. L. Carter, and R. Pochampally, "Serum-deprived human multipotent mesenchymal stromal cells (MSCs) are highly angiogenic," Stem Cell Research, vol. 6, no. 3, pp. 215-225, 2011.

[47] G. E. Kilroy, S. J. Foster, X. Wu et al., "Cytokine profile of human adipose-derived stem cells: expression of angiogenic, hematopoietic, and pro-inflammatory factors," Journal of Cellular Physiology, vol. 212, no. 3, pp. 702-709, 2007.

[48] D. O. Traktuev, S. Merfeld-Clauss, J. Li et al., "A population of multipotent CD34-positive adipose stromal cells share pericyte and mesenchymal surface markers, reside in a periendothelial location, and stabilize endothelial networks," Circulation Research, vol. 102, no. 1, pp. 77-85, 2008.

[49] D. O. Traktuev, D. N. Prater, S. Merfeld-Clauss et al., "Robust functional vascular network formation in vivo by cooperation of adipose progenitor and endothelial cells," Circulation Research, vol. 104, no. 12, pp. 1410-1420, 2009.

[50] L. Li, S. Zhang, Y. Zhang, B. Yu, Y. Xu, and Z. Guan, "Paracrine action mediate the antifibrotic effect of transplanted mesenchymal stem cells in a rat model of global heart failure," Molecular Biology Reports, vol. 36, no. 4, pp. 725-731, 2009.

[51] S. Ohnishi, H. Sumiyoshi, S. Kitamura, and N. Nagaya, "Mesenchymal stem cells attenuate cardiac fibroblast proliferation and collagen synthesis through paracrine actions," FEBS Letters, vol. 581, no. 21, pp. 3961-3966, 2007.

[52] C. Mias, O. Lairez, E. Trouche et al., "Mesenchymal stem cells promote matrix metalloproteinase secretion by cardiac fibroblasts and reduce cardiac ventricular fibrosis after myocardial infarction," Stem Cells, vol. 27, no. 11, pp. 27342743, 2009.

[53] T. B. Rogers, S. Pati, S. Gaa et al., "Mesenchymal stem cells stimulate protective genetic reprogramming of injured cardiac ventricular myocytes," Journal of Molecular and Cellular Cardiology, vol. 50, no. 2, pp. 346-356, 2011.

[54] J. Rehman, D. Traktuev, J. Li et al., "Secretion of angiogenic and antiapoptotic factors by human adipose stromal cells," Circulation, vol. 109, no. 10, pp. 1292-1298, 2004.

[55] S. Aggarwal and M. F. Pittenger, "Human mesenchymal stem cells modulate allogeneic immune cell responses," Blood, vol. 105, no. 4, pp. 1815-1822, 2005.

[56] X. P. Huang, Z. Sun, Y. Miyagi et al., "Differentiation of allogeneic mesenchymal stem cells induces immunogenicity and limits their long-term benefits for myocardial repair," Circulation, vol. 122, no. 23, pp. 2419-2429, 2010.

[57] J. B. Mitchell, K. McIntosh, S. Zvonic et al., "Immunophenotype of human adipose-derived cells: temporal changes in stromal-associated and stem cell-associated markers," Stem Cells, vol. 24, no. 2, pp. 376-385, 2006.
[58] K. McIntosh, S. Zvonic, S. Garrett et al., "The immunogenicity of human adipose-derived cells: temporal changes in vitro," Stem Cells, vol. 24, no. 5, pp. 1246-1253, 2006.

[59] M. J. Crop, C. C. Baan, S. S. Korevaar et al., "Inflammatory conditions affect gene expression and function of human adipose tissue-derived mesenchymal stem cells," Clinical and experimental immunology, vol. 162, no. 3, pp. 474-486, 2010.

[60] K. Takahashi and S. Yamanaka, "Induction of pluripotent stem cells from mouse embryonic and adult fibroblast cultures by defined factors," Cell, vol. 126, no. 4, pp. 663-676, 2006.

[61] P. A. Tat, H. Sumer, K. L. Jones, K. Upton, and P. J. Verma, "The efficient generation of induced pluripotent stem (iPS) cells from adult mouse adipose tissue-derived and neural stem cells," Cell Transplantation, vol. 19, no. 5, pp. 525-536, 2010.

[62] N. Sun, N. J. Panetta, D. M. Gupta et al., "Feeder-free derivation of induced pluripotent stem cells from adult human adipose stem cells," Proceedings of the National Academy of Sciences of the United States of America, vol. 106, no. 37, pp. 15720-15725, 2009.

[63] M. K. Mamidi, R. Pal, N. A.B. Mori et al., "Co-culture of mesenchymal-like stromal cells derived from human foreskin permits long term propagation and differentiation of human embryonic stem cells," Journal of Cellular Biochemistry, vol. 112, no. 5, pp. 1353-1363, 2011.

[64] S. T. Hwang, S. W. Kang, S. J. Lee et al., "The expansion of human ES and iPS cells on porous membranes and proliferating human adipose-derived feeder cells," Biomaterials, vol. 31, no. 31, pp. 8012-8021, 2010.

[65] M. Nian, P. Lee, N. Khaper, and P. Liu, "Inflammatory cytokines and postmyocardial infarction remodeling," Circulation Research, vol. 94, no. 12, pp. 1543-1553, 2004.

[66] D. L. Mann, "Mechanisms and models in heart failure: a combinatorial approach," Circulation, vol. 100, no. 9, pp. 999-1008, 1999.

[67] M. S. Penn, "Importance of the SDF-1: CXCR4 axis in myocardial repair," Circulation Research, vol. 104, no. 10, pp. 1133-1135, 2009.

[68] J. P. M. Cleutjens, J. C. Kandala, E. Guarda, R. V. Guntaka, and K. T. Weber, "Regulation of collagen degradation in the rat myocardium after infarction," Journal of Molecular and Cellular Cardiology, vol. 27, no. 6, pp. 1281-1292, 1995.

[69] M. Ii, M. Horii, A. Yokoyama et al., "Synergistic effect of adipose-derived stem cell therapy and bone marrow progenitor recruitment in ischemic heart," Laboratory Investigation, vol. 91, no. 4, pp. 539-552, 2011.

[70] R. Gaebel, D. Furlani, H. Sorg et al., "Cell origin of human mesenchymal stem cells determines a different healing performance in cardiac regeneration," PLoS ONE, vol. 6, no. 2, Article ID e15652, 2011.

[71] X. Bai, Y. Yan, M. Coleman et al., "Tracking long-term survival of intramyocardially delivered human adipose tissuederived stem cells using bioluminescence imaging," Molecular Imaging and Biology, pp. 1-13, 2010.

[72] C. Dubois, X. Liu, P. Claus et al., "Differential Effects of Progenitor Cell Populations on Left Ventricular Remodeling and Myocardial Neovascularization After Myocardial Infarction," Journal of the American College of Cardiology, vol. 55, no. 20, pp. 2232-2243, 2010.

[73] Y. J. Yang, H. Y. Qian, J. Huang et al., "Combined therapy with simvastatin and bone marrow-derived mesenchymal stem cells increases benefits in infarcted swine hearts," 
Arteriosclerosis, Thrombosis, and Vascular Biology, vol. 29, no. 12, pp. 2076-2082, 2009.

[74] K. E. A. van der Bogt, S. Schrepfer, J. Yu et al., "Comparison of transplantation of adipose tissue- and bone marrowderived mesenchymal stem cells in the infarcted heart," Transplantation, vol. 87, no. 5, pp. 642-652, 2009.

[75] J. A. Dixon, R. C. Gorman, R. E. Stroud et al., "Mesenchymal cell transplantation and myocardial remodeling after myocardial infarction," Circulation, vol. 120, no. 1, pp. S220S229, 2009.

[76] X. Bai, Y. Yan, Y. H. Song et al., "Both cultured and freshly isolated adipose tissue-derived stem cells enhance cardiac function after acute myocardial infarction," European Heart Journal, vol. 31, no. 4, pp. 489-501, 2010.

[77] K. E. van der Bogt, A. Y. Sheikh, S. Schrepfer et al., "Comparison of different adult stem cell types for treatment of myocardial ischemia," Circulation, vol. 118, no. 14, pp. S121-129, 2008.

[78] Y. Imanishi, A. Saito, H. Komoda et al., "Allogenic mesenchymal stem cell transplantation has a therapeutic effect in acute myocardial infarction in rats," Journal of Molecular and Cellular Cardiology, vol. 44, no. 4, pp. 662-671, 2008.

[79] S. M. Hashemi, S. Ghods, F. D. Kolodgie et al., "A placebo controlled, dose-ranging, safety study of allogenic mesenchymal stem cells injected by endomyocardial delivery after an acute myocardial infarction," European Heart Journal, vol. 29, no. 2, pp. 251-259, 2008.

[80] S. L. Hale, W. Dai, J. S. Dow, and R. A. Kloner, "Mesenchymal stem cell administration at coronary artery reperfusion in the rat by two delivery routes: a quantitative assessment," Life Sciences, vol. 83, no. 13-14, pp. 511-515, 2008.

[81] C. A. Carr, D. J. Stuckey, L. Tatton et al., "Bone marrowderived stromal cells home to and remain in the infarcted rat heart but fail to improve function: an in vivo cine-MRI study," American Journal of Physiology, Heart and Circulatory Physiology, vol. 295, no. 2, pp. H533-H542, 2008.

[82] L. Cai, B. H. Johnstone, T. G. Cook et al., "IFATS collection: human adipose tissue-derived stem cells induce angiogenesis and nerve sprouting following myocardial infarction, in conjunction with potent preservation of cardiac function," Stem Cells, vol. 27, no. 1, pp. 230-237, 2008.

[83] C. Valina, K. Pinkernell, Y. H. Song et al., "Intracoronary administration of autologous adipose tissue-derived stem cells improves left ventricular function, perfusion, and remodelling after acute myocardial infarction," European Heart Journal, vol. 28, no. 21, pp. 2667-2677, 2007.

[84] B. Li, Q. Zeng, H. Wang et al., "Adipose tissue stromal cells transplantation in rats of acute myocardial infarction," Coronary Artery Disease, vol. 18, no. 3, pp. 221-227, 2007.

[85] J. I. Virag and C. E. Murry, "Myofibroblast and endothelial cell proliferation during murine myocardial infarct repair," American Journal of Pathology, vol. 163, no. 6, pp. 2433-2440, 2003.

[86] Y. Sun, M. F. Kiani, A. E. Postlethwaite, and K. T. Weber, "Infarct scar as living tissue," Basic Research in Cardiology, vol. 97, no. 5, pp. 343-347, 2002.

[87] H. Song, M. J. Cha, B. W. Song et al., "Reactive oxygen species inhibit adhesion of mesenchymal stem cells implanted into ischemic myocardium via interference of focal adhesion complex," Stem Cells, vol. 28, no. 3, pp. 555-563, 2010.

[88] M. Mazo, J. J. Gavira, G. Abizanda et al., "Transplantation of mesenchymal stem cells exerts a greater long-term effect than bone marrow mononuclear cells in a chronic myocardial infarction model in rat," Cell Transplantation, vol. 19, no. 3, pp. 313-328, 2009.

[89] M. F. Chen, B. C. Lee, H. C. Hsu et al., "Cell therapy generates a favourable chemokine gradient for stem cell recruitment into the infarcted heart in rabbits," European Journal of Heart Failure, vol. 11, no. 3, pp. 238-245, 2009.

[90] M. Mazo, V. Planat-Bénard, G. Abizanda et al., "Transplantation of adipose derived stromal cells is associated with functional improvement in a rat model of chronic myocardial infarction," European Journal of Heart Failure, vol. 10, no. 5, pp. 454-462, 2008.

[91] K. Jujo, M. Ii, and D. W. Losordo, "Endothelial progenitor cells in neovascularization of infarcted myocardium," Journal of Molecular and Cellular Cardiology, vol. 45, no. 4, pp. 530544, 2008.

[92] M. Nahrendorf, F. K. Swirski, E. Aikawa et al., "The healing myocardium sequentially mobilizes two monocyte subsets with divergent and complementary functions," Journal of Experimental Medicine, vol. 204, no. 12, pp. 3037-3047, 2007.

[93] L. Wang, J. Deng, W. Tian et al., "Adipose-derived stem cells are an effective cell candidate for treatment of heart failure: an MR imaging study of rat hearts," American Journal of Physiology, Heart and Circulatory Physiology, vol. 297, no. 3, pp. H1020-H1031, 2009.

[94] L. C. Amado, A. P. Saliaris, K. H. Schuleri et al., "Cardiac repair with intramyocardial injection of allogeneic mesenchymal stem cells after myocardial infarction," Proceedings of the National Academy of Sciences of the United States of America, vol. 102, no. 32, pp. 11474-11479, 2005.

[95] H. Li, D. Malhotra, C. C. Yeh et al., "Myocardial survival signaling in response to stem cell transplantation," Journal of the American College of Surgeons, vol. 208, no. 4, pp. 607-613, 2009.

[96] J. Cho, P. Zhai, Y. Maejima, and J. Sadoshima, "Myocardial injection with GSK-3 $\beta$-overexpressing bone marrow-derived mesenchymal stem cells attenuates cardiac dysfunction after myocardial infarction," Circulation Research, vol. 108, no. 4, pp. 478-489, 2011.

[97] X. Wang, T. Zhao, W. Huang et al., "Hsp20-engineered mesenchymal stem cells are resistant to oxidative stress via enhanced activation of Akt and increased secretion of growth factors," Stem Cells, vol. 27, no. 12, pp. 3021-3031, 2009.

[98] K. Le Blanc, "Mesenchymal stromal cells: tissue repair and immune modulation," Cytotherapy, vol. 8, no. 6, pp. 559-561, 2006.

[99] K. H. Schuleri, L. C. Amado, A. J. Boyle et al., "Early improvement in cardiac tissue perfusion due to mesenchymal stem cells," American Journal of Physiology, Heart and Circulatory Physiology, vol. 294, no. 5, pp. H2002-H2011, 2008.

[100] L. C. Amado, K. H. Schuleri, A. P. Saliaris et al., "Multimodality noninvasive imaging demonstrates in vivo cardiac regeneration after mesenchymal stem cell therapy," Journal of the American College of Cardiology, vol. 48, no. 10, pp. 21162124, 2006.

[101] M. Rigol, N. Solanes, J. Farré et al., "Effects of adipose tissuederived stem cell therapy after myocardial infarction: impact of the route of administration," Journal of Cardiac Failure, vol. 16, no. 4, pp. 357-366, 2010.

[102] Y. Zhou, S. Wang, Z. Yu et al., "Direct injection of autologous mesenchymal stromal cells improves myocardial function," Biochemical and Biophysical Research Communications, vol. 390, no. 3, pp. 902-907, 2009.

[103] J. Ma, J. Ge, S. Zhang et al., "Time course of myocardial stromal cell-derived factor 1 expression and beneficial effects 
of intravenously administered bone marrow stem cells in rats with experimental myocardial infarction," Basic Research in Cardiology, vol. 100, no. 3, pp. 217-223, 2005.

[104] Z. Cheng, L. Ou, X. Zhou et al., "Targeted migration of mesenchymal stem cells modified with CXCR4 gene to infarcted myocardium improves cardiac performance," Molecular Therapy, vol. 16, no. 3, pp. 571-579, 2008.

[105] Z. Cheng, X. Liu, L. Ou et al., "Mobilization of mesenchymal stem cells by granulocyte colony-stimulating factor in rats with acute myocardial infarction," Cardiovascular Drugs and Therapy, vol. 22, no. 5, pp. 363-371, 2008.

[106] J. Huang, Z. Zhang, J. Guo et al., "Genetic modification of mesenchymal stem cells overexpressing ccr1 increases cell viability, migration, engraftment, and capillary density in the injured myocardium," Circulation Research, vol. 106, no. 11, pp. 1753-1762, 2010.

[107] X. H. Liu, C. G. Bai, Z. Y. Xu et al., "Therapeutic potential of angiogenin modified mesenchymal stem cells: angiogenin improves mesenchymal stem cells survival under hypoxia and enhances vasculogenesis in myocardial infarction," Microvascular Research, vol. 76, no. 1, pp. 23-30, 2008.

[108] B. Zeng, H. Chen, C. Zhu, X. Ren, G. Lin, and F. Cao, "Effects of combined mesenchymal stem cells and heme oxygenase1 therapy on cardiac performance," European Journal of Cardio-Thoracic Surgery, vol. 34, no. 4, pp. 850-856, 2008.

[109] M. Gnecchi, H. He, L. G. Melo et al., "Early beneficial effects of bone marrow-derived mesenchymal stem cells overexpressing akt on cardiac metabolism after myocardial infarction," Stem Cells, vol. 27, no. 4, pp. 971-979, 2009.

[110] M. Gnecchi, H. He, N. Noiseux et al., "Evidence supporting paracrine hypothesis for Akt-modified mesenchymal stem cell-mediated cardiac protection and functional improvement," FASEB Journal, vol. 20, no. 6, pp. 661-669, 2006.

[111] M. Gnecchi, H. He, O. D. Liang et al., "Paracrine action accounts for marked protection of ischemic heart by Aktmodified mesenchymal stem cells," Nature Medicine, vol. 11, no. 4, pp. 367-368, 2005.

[112] H. Song, M. J. Cha, B. W. Song et al., "Reactive oxygen species inhibit adhesion of mesenchymal stem cells implanted into ischemic myocardium via interference of focal adhesion complex," Stem Cells, vol. 28, no. 3, pp. 555-563, 2010.

[113] J. Jin, S. I. Jeong, Y. M. Shin et al., "Transplantation of mesenchymal stem cells within a poly(lactide-co- $\varepsilon$ - caprolactone) scaffold improves cardiac function in a rat myocardial infarction model," European Journal of Heart Failure, vol. 11, no. 2, pp. 147-153, 2009.

[114] M. Y. Tan, W. Zhi, R. Q. Wei et al., "Repair of infarcted myocardium using mesenchymal stem cell seeded small intestinal submucosa in rabbits," Biomaterials, vol. 30, no. 19, pp. 3234-3240, 2009.

[115] C. H. Chen, H. J. Wei, W. W. Lin et al., "Porous tissue grafts sandwiched with multilayered mesenchymal stromal cell sheets induce tissue regeneration for cardiac repair," Cardiovascular Research, vol. 80, no. 1, pp. 88-95, 2008.

[116] Y. Miyahara, N. Nagaya, M. Kataoka et al., "Monolayered mesenchymal stem cells repair scarred myocardium after myocardial infarction," Nature Medicine, vol. 12, no. 4, pp. 459-465, 2006.

[117] A. Bel, V. Planat-Bernard, A. Saito et al., "Composite cell sheets: a further step toward safe and effective myocardial regeneration by cardiac progenitors derived from embryonic stem cells," Circulation, vol. 122, no. 11, pp. S118-S123, 2010.

[118] S. Chen, Z. Liu, N. Tian et al., "Intracoronary transplantation of autologous bone marrow mesenchymal stem cells for ischemic cardiomyopathy due to isolated chronic occluded left anterior descending artery," Journal of Invasive Cardiology, vol. 18, no. 11, pp. 552-556, 2006.

[119] D. G. Katritsis, P. A. Sotiropoulou, E. Karvouni et al., "Transcoronary transplantation of autologous mesenchymal stem cells and endothelial progenitors into infarcted human myocardium," Catheterization and Cardiovascular Interventions, vol. 65, no. 3, pp. 321-329, 2005.

[120] S. L. Chen, W. W. Fang, F. Ye et al., "Effect on left ventricular function of intracoronary transplantation of autologous bone marrow mesenchymal stem cell in patients with acute myocardial infarction," American Journal of Cardiology, vol. 94, no. 1, pp. 92-95, 2004.

[121] J. M. Hare, "Translational development of mesenchymal stem cell therapy for cardiovascular diseases," Texas Heart Institute Journal, vol. 36, no. 2, pp. 145-147, 2009.

[122] J. M. Hare, J. H. Traverse, T. D. Henry et al., "A randomized, double-blind, placebo-controlled, dose-escalation study of intravenous adult human mesenchymal stem cells (prochymal) after acute myocardial infarction," Journal of the American College of Cardiology, vol. 54, no. 24, pp. 22772286, 2009.

[123] N. Dib, T. Henry, A. DeMaria, S. Itescu, M. M. McCarthy, and S. C. Jaggar, "The first US study to assess the feasibility and safety of endocardial delivery of allogenic mesenchymal precursor cells in patient with heart failure: three-month interim analysis," Circulation, vol. 120, p. S810, 2009.

[124] H. J. Duckers, J. Houtgraaf, R. J. van Geuns, B. D. van Dalen, E. Regar, and W. van der Giessen, "First-in-man experience with intracoronary infusion of adipose-derived regenerative cells in the treatment of patients with ST-elevation myocardial infarction: the apollo trial," Circulation, vol. 120, Article ID A12225, 2010.

[125] A. Schaefer, C. Zwadlo, M. Fuchs et al., "Long-term effects of intracoronary bone marrow cell transfer on diastolic function in patients after acute myocardial infarction: 5-year results from the randomized-controlled BOOST trial-an echocardiographic study," European Journal of Echocardiography, vol. 11, no. 2, pp. 165-171, 2010.

[126] J. A. Miettinen, K. Ylitalo, P. Hedberg et al., "Determinants of functional recovery after myocardial infarction of patients treated with bone marrow-derived stem cells after thrombolytic therapy," Heart, vol. 96, no. 5, pp. 362-367, 2009.

[127] C. Stamm, H. D. Kleine, Y. H. Choi et al., "Intramyocardial delivery of CD133+ bone marrow cells and coronary artery bypass grafting for chronic ischemic heart disease: safety and efficacy studies," Journal of Thoracic and Cardiovascular Surgery, vol. 133, no. 3, pp. 717-725, 2007.

[128] E. Martin-Rendon, S. J. Brunskill, C. J. Hyde, S. J. Stanworth, A. Mathur, and S. M. Watt, "Autologous bone marrow stem cells to treat acute myocardial infarction: a systematic review," European Heart Journal, vol. 29, no. 15, pp. 18071818, 2008.

[129] T. S. Li, M. Kubo, K. Ueda, M. Murakami, A. Mikamo, and K. Hamano, "Impaired angiogenic potency of bone marrow cells from patients with advanced age, anemia, and renal failure," Journal of Thoracic and Cardiovascular Surgery, vol. 139, no. 2, pp. 459-465, 2010.

[130] C. K. Kissel, R. Lehmann, B. Assmus et al., "Selective functional exhaustion of hematopoietic progenitor cells in the bone marrow of patients with postinfarction heart failure," Journal of the American College of Cardiology, vol. 49, no. 24, pp. 2341-2349, 2007. 
[131] S. A. Sorrentino, F. H. Bahlmann, C. Besler et al., "Oxidant stress impairs in vivo reendothelialization capacity of endothelial progenitor cells from patients with type 2 diabetes mellitus: restoration by the peroxisome proliferatoractivated receptor- $\gamma$ agonist rosiglitazone," Circulation, vol. 116, no. 2, pp. 163-173, 2007.

[132] A. J. Nauta and W. E. Fibbe, "Immunomodulatory properties of mesenchymal stromal cells," Blood, vol. 110, no. 10, pp. 3499-3506, 2007.

[133] J. C. Chachques, J. C. Trainini, N. Lago et al., "Myocardial assistance by grafting a new bioartificial upgraded myocardium (MAGNUM clinical trial): one year follow-up," Cell Transplantation, vol. 16, no. 9, pp. 927-934, 2007. 

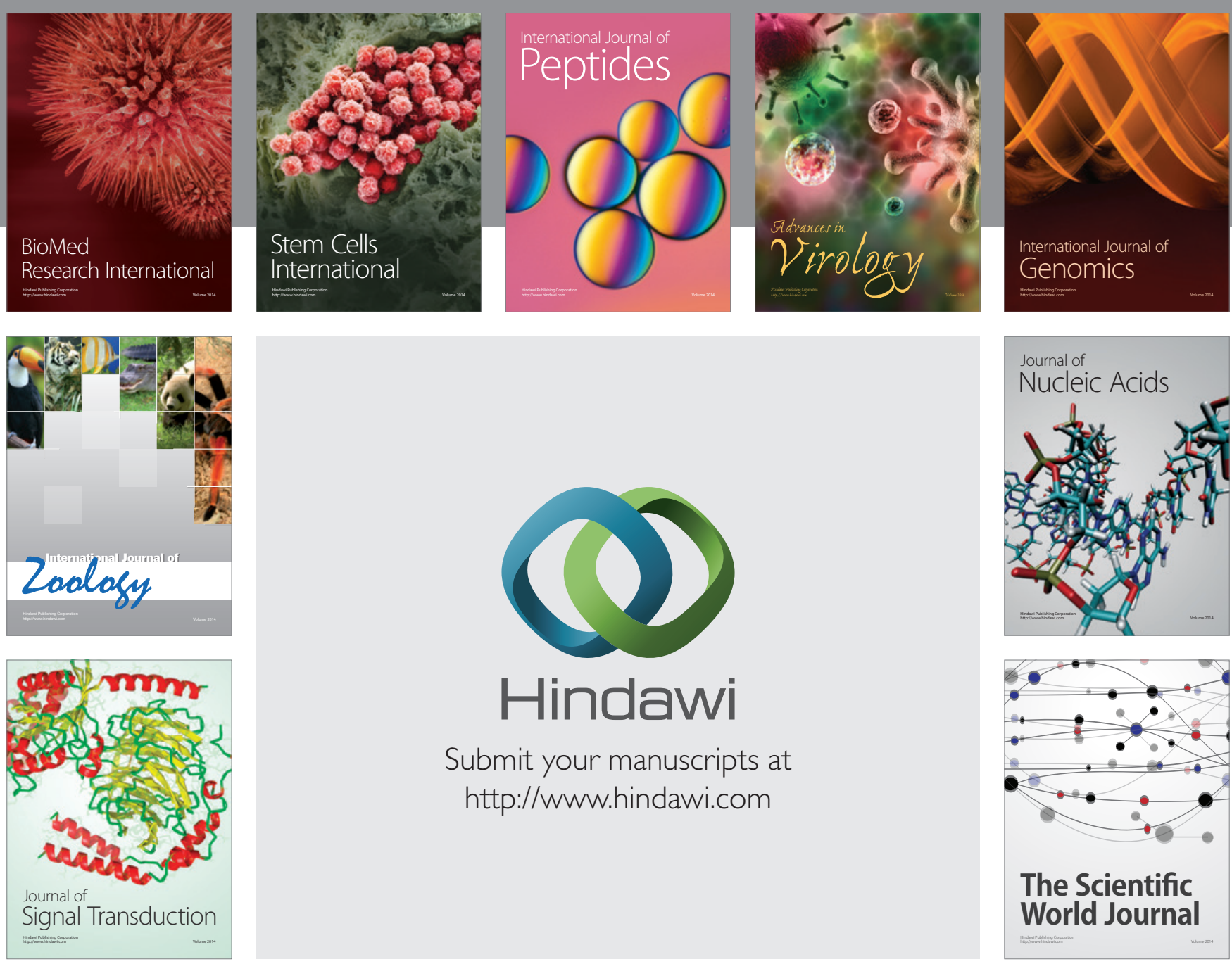

Submit your manuscripts at

http://www.hindawi.com
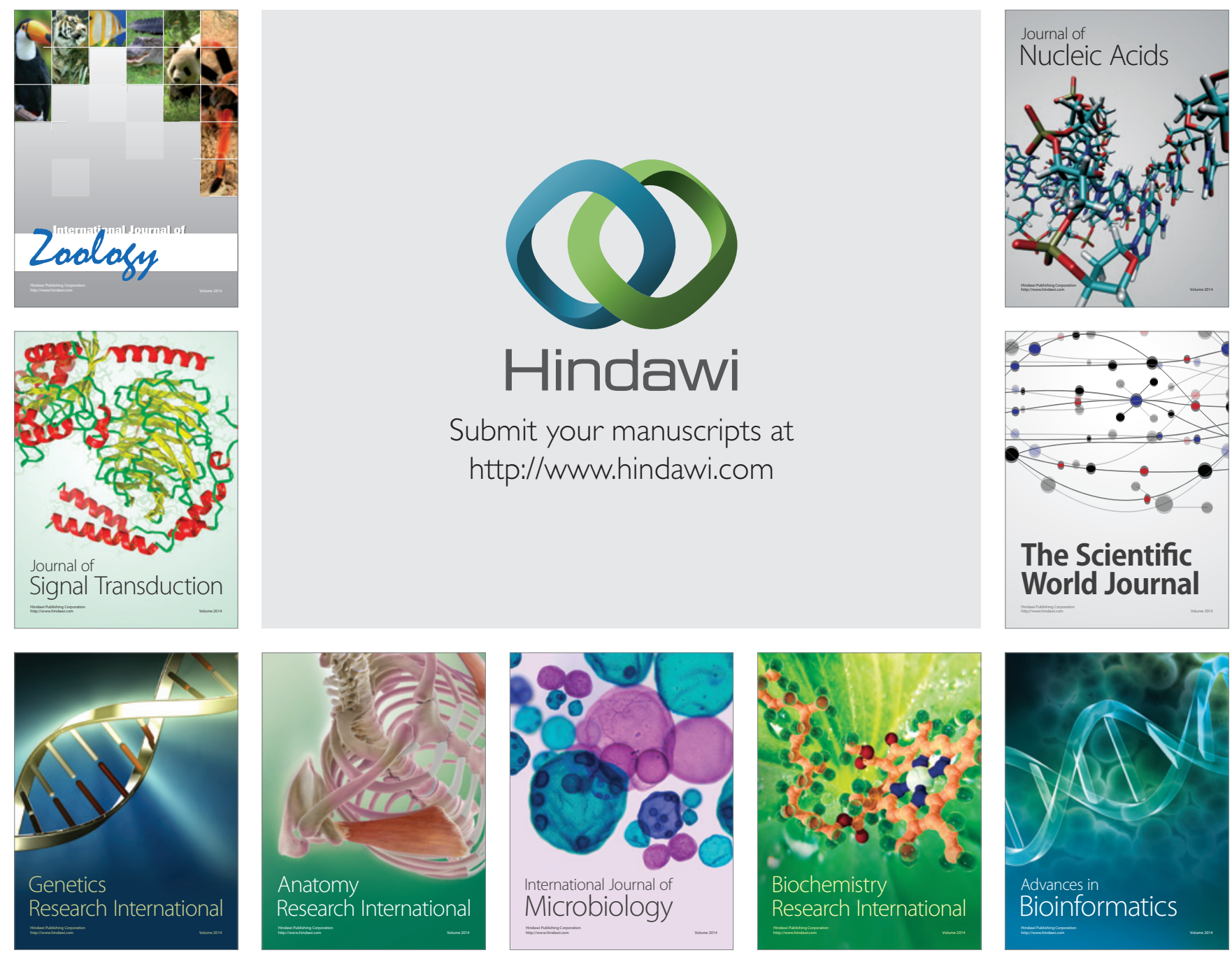

The Scientific World Journal
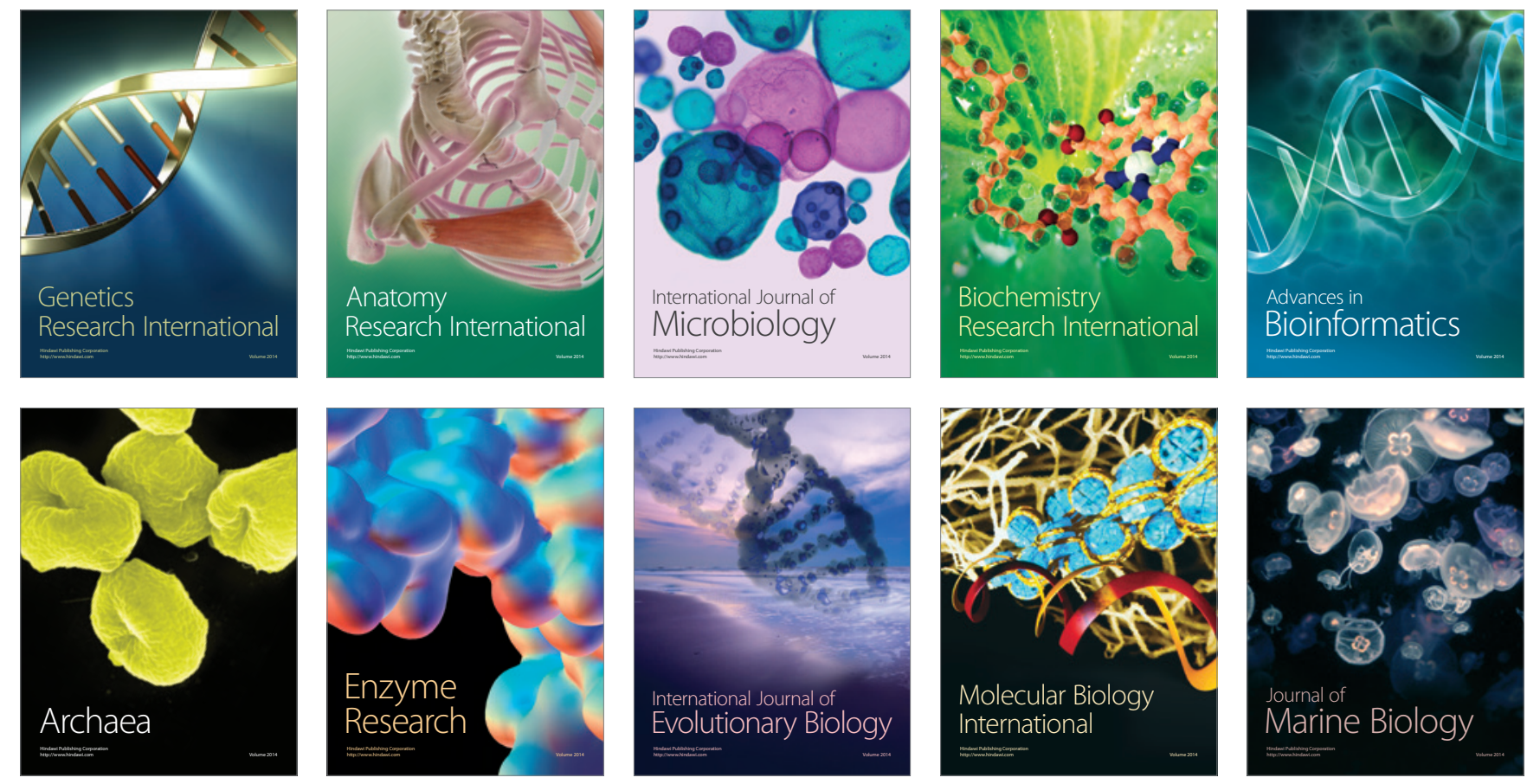